\title{
DESIGNING QUESTIONNAIRES FOR NUTRITION RESEARCH
}

\author{
A. M. Fehily \& A. P. Johns
}

\section{INTRODUCTION}

Questionnaires are used in most research studies to record basic information about the subjects such as date of birth and sex, to record measurements such as height and weight, or to collect information such as smoking habit, medical history, attitudes or food intake. When developing a questionnaire, there are a large number of factors that need to be considered at the design stage, including how data will be processed. This involves a lot of work, but is essential to ensure the effectiveness and efficiency of data collection and analysis. If adequate consideration is not given to the design of forms and coding systems for example, the result may be weeks of additional data processing time or, in the worst case, collected data may not be able to be used as originally envisaged.

The table below shows the main factors that need to be considered when developing a questionnaire. Some of these are relevant only to questionnaires to assess food and nutrient intakes, but most are relevant to all questionnaires. These factors are discussed in the following sections, providing guidance to help overcome many of the potential pitfalls in data collection.

\begin{tabular}{|l|}
\hline Factors to be Considered when Developing a Questionnaire \\
\hline \\
\hline The nutrient(s) to be assessed \\
\hline The foods in which the nutrient(s) is present \\
\hline Food portion sizes \\
\hline Method of administration: \\
self-administered or interviewer-administered \\
\hline $\begin{array}{l}\text { Media to be used: } \\
\text { paper form or computerised interview }\end{array}$ \\
\hline Location of questionnaire completion: \\
at home, in a clinic or by telephone \\
\hline Question format: open or closed \\
\hline Length of questionnaire \\
\hline Sequence of questions \\
\hline Wording of questions \\
\hline Coding of data \\
\hline Range \& compatibility checks on data \\
\hline Calculation of nutrient intakes \\
\hline Missing values processing \\
\hline Pilot study \\
\hline Validation of intake data \\
\hline
\end{tabular}

\section{NUTRIENTS AND FOODS}

If the questionnaire is to be used to estimate nutrient intakes, the first consideration is which nutrients are to be assessed and whether a questionnaire is an appropriate method for these nutrients.

Food intake questionnaires have been widely used in large-scale population surveys. They have several advantages over other dietary survey methods. Firstly, data can be collected quickly and cheaply. Data can be collected from several geographical areas, the forms being returned by post. Observer bias is minimised. In addition, data are obtained in a form convenient for coding and statistical analysis. The dietary information tends to be less precise than that obtained in other ways. However, it may be more representative of people's usual intake than that derived from a detailed study of a few days' food consumption. Some nutrients are much easier to study in this way than others. For example, intake of 
fibre or vitamin $C$ can be more readily estimated by questionnaire than, say, fat intake. This is because fibre and vitamin $C$ are present in relatively few foods and people can estimate how often they eat these foods reasonably well. Day to day variation in intake of a nutrient is also a factor in how well that nutrient can be estimated by questionnaire - in general, intakes of nutrients that do not vary greatly from day to day can be more readily estimated than those for which there is large day to day variation.

A list of all the nutrients of interest should be made initially. This is important as a questionnaire designed to assess, say, fibre and sugar intakes could not be used at a later date to calculate intakes of other nutrients if food sources of those nutrients are not the same, e.g. fat.

The food sources of each nutrient within the population being studied should then be considered. Criteria for the selection of foods are that they should be eaten reasonably often by an appreciable number of individuals and that they should have a substantial content of the nutrient(s) of interest. If the purpose of the survey requires that subjects be classified into tertiles or quintiles according to their nutrient intakes then, in order to differentiate between individuals, foods should be included for which there is likely to be reasonable variation in consumption between individuals. If the questionnaire is to be used to estimate mean nutrient intakes of the population, or to compare the mean intakes of subgroups, the foods selected for the questionnaire should cover at least $90-95 \%$ of each nutrient to be calculated. If a lower proportion of the total intake is covered then group mean intakes may be substantially underestimated.

Consideration should be given to the grouping of foods within the questionnaire. For example, should "tomatoes" be asked in a separate question or should this be included with "salads"? Should a question be asked about "beef, all forms including minced beef" or should there be a number of questions: "roast beef", "beef stew", "minced beef", "beef burgers" etc.? Appropriate groupings will be dependent upon a number of factors: the hypotheses being tested; how similar the nutrient compositions of the foods are; and the likely ability of subjects to assess how often they eat the foods. For example, people may be better able to estimate how often they have "meals containing beef" than to estimate separately the frequency of consumption for each form of beef. Another consideration is whether subjects may count the same food twice when completing the questionnaire. For example, if there is a question about "beef lasagne" and another question about "pasta", does the latter include or exclude the pasta accounted for in "beef lasagne"?

\section{FOOD PORTION SIZES}

If the questionnaire is to be used to calculate nutrient intakes, it is necessary to decide whether to use average portion weights, to differentiate between small medium and large portions, or to assess portion sizes for every individual. For some foods, subjects may be able to report portion sizes relatively easily, e.g. the number and size of slice for bread. An average value could then be used for each slice size. For some foods, such as eggs or apples, questions could be included about the number eaten per week. In this case an average value could be used for one apple or one egg. For other foods, such as meats, rice and pasta which do not come in "units", there are a number of options. For these foods, an average portion weight could be used (Bingham \& Day, 1987; Fehily \& Hopkinson, 1993; Crawley,1994) or one could assess portion sizes by means of descriptions, photographs or food models (Crawley, 1994; Nelson et al, 1997). However, portion sizes for many foods vary considerably within an individual over time and this variation has been found to be greater than that between persons of similar age and sex (Hunter et al, 1988).

Studies investigating the effect of portion size estimates on the ability to rank individuals by their nutrient intake have shown very high correlations $(r=0.9)$ between intake estimates using average portion sizes and those using assessed or weighed portion sizes (Willett, 1990; Fehily \& Hopkinson, 1993). It has also been reported that most of the variation in intake of any food is explained by frequency of consumption (Willett, 1990). The use of average portion sizes is therefore likely to have a minimal effect on the nutrient intake estimates from a food frequency questionnaire. 


\section{METHOD OF ADMINISTRATION}

One needs to decide whether the questionnaire will be administered by an interviewer or selfadministered. An advantage of a self-administered questionnaire is that it can be sent by post and returned in a prepaid envelope. The survey can therefore cover a wide geographical area. The fact that interviewers are not required reduces the need for training and resources and minimises observer bias. However, it is important to check the questionnaires on return as some answers may be missing or ambiguous.

If the questionnaire is to be interviewer-administered, then there will be a need for training. This is necessary to minimise both intra- and inter-interviewer variation. A common fault in intervieweradministered questionnaires is that of suggesting answers to the subjects. It is very important that the questionnaire is administered in the same way by all interviewers if there is more than one interviewer. An additional problem is that subjects may tell the interviewer what they think he/she wishes to know rather than the true answer. Intervention studies where compliance with dietary advice is being assessed may be particularly prone to this, but the extent of the problem will depend upon the rapport between the subject and the interviewer. Use of an interviewer-administered questionnaire requires more resources than a self-administered questionnaire, but it will reduce the likelihood of incomplete or ambiguous answers.

\section{MEDIA USED}

The questionnaire may be either a paper form or a computerised interview. The use of a paper form means that it could be sent by post or given to subjects for completion at home. However, the disadvantage is that the form requires coding, i.e. conversion of responses to numerical or other symbols for subsequent computer analysis. This takes time, even if the coding scheme is simple. The coding must be checked and data then entered into a computer. Data entry should be done twice (dual data entry with computerised verification check) to reduce the possibility of errors in data entry. Nevertheless, poor handwriting can still result in errors - a figure may be read wrongly twice!

A computerised interview could be done by the subjects themselves, depending upon the complexity of the questionnaire, or by an interviewer. If done by an interviewer, it could be done in a clinic, in a subject's home or by telephone. A major advantage of a computerised interview is that the need for coding of data, checking of coding and subsequent data entry is eliminated. An additional advantage is that responses can be range checked during the interview. This reduces the potential for gross errors. In addition, if the questionnaire is concerned with food intake, range checks can be made on the responses, thereby helping to ensure that the overall diet is "reasonable".

\section{LOCATION OF COMPLETION}

Is the questionnaire to be completed at home, in a clinic or by telephone? If the questionnaire is to be completed at home, this has the advantage that there is more time to complete it than in a clinic or by telephone.

In a clinic situation, the time available to complete the questionnaire may be limited, being dependent upon what other questionnaires and examinations are to be completed during the same time period. If dealing with middle-aged or elderly subjects, it is advisable to warn them beforehand if they are to complete the questionnaire themselves, otherwise they may forget to bring reading glasses with them. It is also advisable to ensure that someone is available to help those who are unable to complete the questionnaire themselves. If subjects are not normally involved in shopping and cooking, they may not be able to adequately answer questions about their food intake. In this case, it may be better if the questionnaire is completed at home, with the person responsible for shopping and cooking.

If the questionnaire is to be completed by telephone, it is advisable to establish at the beginning of the interview that the call is at a convenient time and to advise each subject how long the interview is likely to take. This may help to reduce the potential for ill-considered answers if the subject does not have sufficient time to complete the interview comfortably. 


\section{QUESTION FORMAT}

Is the question format to be open or closed? Closed format is where the response is one or more selections from a list, or a number within a pre-defined range. Open format is where the response cannot be pre-determined, e.g., free-text, such as an opinion or "other comments". For a computerised interview, the questions need to be closed format. For a paper form, it needs to be borne in mind that open format questions are more difficult to handle in terms of coding. It is therefore advisable to use closed format questions wherever possible.

The questionnaire needs to contain an instruction as to how it should be completed, together with an example. On a paper form, the clearest method is to ask subjects to ring the appropriate answer, for example, in questions concerning the frequency of consumption of a range of foods. There are potential problems with other methods of indicating an answer from a list. For example, if there are only two alternative answers to a question, a tick or cross may indicate that this is the intended answer, or it may indicate a deleted response such that the true answer is the unmarked one. If subjects are asked to write a number in a box to indicate say, the frequency of consumption, they need to remember which number relates to each frequency. This may be straightforward if the response "three days/week" is to be indicated by " 3 " and "everyday" is to be indicated by " 7 ". However, it may be confusing if "three servings/week " is to be indicated by " 3 " but "one serving/day" is to be indicated by " 5 ".

The range of responses for each question type should be consistent throughout the questionnaire. For example, if frequency responses used for one question are 1,2,3,4,5,6,7 to indicate the number of days per week, $\mathrm{F}$ to indicate once every 2-3 weeks and $\mathrm{R}$ to indicate rarely or never, then responses to other frequency questions should be the same. The range of responses for each question needs to be adequate to differentiate between individuals. For example, in questions about attitudes, four alternatives are better than five - if five alternatives are given subjects may be most likely to choose the middle (least

informative) response. The type of response may also need to be different for different questions. For example, frequency responses may be appropriate for consumption of foods such as meats, fish, pasta and rice, but not for sugar in tea and coffee, as the range of responses would need to be much greater. In the latter case, it would be better to ask how many cups of each drink are usually consumed per day and how many teaspoons of sugar are added to each cup.

\section{LENGTH OF QUESTIONNAIRE}

If the questionnaire is too long, subjects may refuse to complete it. This is an important factor in any population survey, as a high response rate is essential. If the response rate is not high, subjects who take part in the study cannot be said to be representative of the population from which they were selected. As a guideline, a single questionnaire should not take longer than about 20 minutes to complete to minimise the potential for a detrimental effect on response rate.

If the questionnaire is very long, subjects may become fatigued or bored, impairing concentration and the accuracy of responses (Willett, 1990). Additional problems with a long questionnaire are that if using a paper form that contains many pages, subjects may turn over two pages together and hence miss out a number of questions. Also, if questions are squashed together in an attempt to reduce the number of pages, subjects may not be able to read it or they may miss questions. As a guideline, for subjects with normal sight it is not advisable to use a point size of less than six. If the survey includes middle-aged or elderly subjects, a point size of less than 10 is not advisable.

\section{SEQUENCE \& WORDING OF QUESTIONS}

The first question should be an easy one. Its meaning should not only be clear but people should be able to answer it. For example, most people will be able to estimate how many slices of bread they eat per day or how often they eat a breakfast cereal, but may not find it so easy to answer how often they eat beef. Hence, a question about bread or breakfast cereal would be a better first question than one about beef. 
The wording used should be simple and with an uncomplicated sentence structure. Questions should be unambiguous. The layout of the paper form is also important. A neat and logically ordered form will help to minimise recording errors.

If the questionnaire is to be self-administered, the language used may need consideration. For those subjects unable to read and write in English, can the questionnaire be made available in other languages?

\section{CODING OF DATA}

Questionnaire responses need to be converted to numerical or other symbols in order for data to be analysed by computer. Instructions for coding should be written as the questionnaire is being developed. The coding scheme should be as simple as possible and consistent rules should be followed. For the coding scheme, numbers are preferable to letters as data are easier to handle in computer analyses and require less storage space.

If a paper form is used, the inclusion of a column of boxes on the right hand side of the form headed "for office use" will enable coding of the information to be carried out easily and quickly. To use the same form for data collection and coding minimises transcription errors. The size of the coding boxes should be large enough such that coded data are legible. Using a consistent coding scheme minimises coding errors. For example, if the answers "no" and "yes" to one question are coded 0 and 1 respectively, then all similar questions should be coded in the same way. None of the coding boxes should be left blank. Thus, there should be a missing value code for every item and a "not applicable" code where appropriate. For example, if a subject never eats a breakfast cereal, a question on the type of cereal is not applicable, but a code is needed for "type of cereal" so that the coding box is not left blank.

\section{RANGE \& COMPATIBILITY CHECKS ON DATA}

Every coded form should be checked for completeness and for gross errors before being entered into a computer. This is, however, only a first step. Rules need to be developed so that computer software can be used to carry out detailed range and compatibility checks to pick out illegal, unlikely and contradictory data.

If a computerised interview is used, coding of data is done automatically, but range and compatibility checks are still required.

\section{CALCULATION OF NUTRIENT INTAKES}

How food consumption data are to be converted to nutrient intakes is an important consideration. Firstly, a nutrient composition must be allocated to each item on the questionnaire. This could be either a single food or average of several foods. For example, if the question is about "beef", which cut of beef is used? Should nutrient values for "lean only" or "lean plus fat" be used? If nutrient values for several foods are to be used for one item on the questionnaire, should average nutrition values be taken for these, or should the nutrient composition values be weighted in some way? The most appropriate food(s) and weighting of the nutrient compositions will be dependent upon the dietary habits of the population being studied.

The nutrient composition used for an item could be modified depending upon the answer to another question. In the beef example above, if subjects are asked whether they usually eat the fat on meat, the composition of "lean+fat" cuts could be used for those who answer "yes" and the composition of "lean only" cuts could be used for those who answer "no". The nutrient composition used for items such as fried foods or home made cakes and pastries could be modified depending upon the answers to other questions about the type and brand of fat used.

For each item on the questionnaire, the frequency of consumption is multiplied by the portion weight to yield a weight of each foodstuff consumed per week. This is then used with the nutrient composition of the item to calculate the mean daily nutrient intakes. 


\section{MISSING VALUES PROCESSING}

For some questionnaires, responses may be incomplete and therefore coded as missing values. This is most likely to be a problem in surveys where a paper form is used and the questionnaire is selfadministered. If the questionnaire is to be used to estimate nutrient intakes, instructions must be standardised as to whether intake of a particular nutrient should still be calculated when some responses are missing. This will be dependent upon the proportion of the total nutrient intake provided by the missing item in the population being studied. For example, sugar intake should not be calculated for a particular individual if it was not known whether sugar is added to tea or coffee. However, if this were the only missing data on that subject's questionnaire, intakes of other nutrients such as fibre could still be calculated.

If the questionnaire is to be used to calculate nutrient intakes, one needs also to consider how missing values are to be processed. For example, if the size of slice for bread is missing, should bread be omitted from the nutrient intake calculation? Or, should nutrition analysis not be done at all for that questionnaire? Or, is it possible to make an assumption about the size of slice if the frequency and number of slices are known? Each option may have a detrimental effect on the overall quality of the survey data, depending upon which data are missing and the number of questionnaires affected. If the missing items are omitted from the nutrition analysis, nutrient intakes will be underestimated. If the whole questionnaire is omitted from the analysis then the response rate of the survey is reduced and the remaining subjects may not be representative of the population from which they were selected. If assumptions made about missing values are inappropriate for the population being studied, calculated mean intakes may still not be an adequate estimate for that population. The best option for each missing value on the questionnaire therefore needs to be given very careful consideration.

\section{PILOT STUDY}

Having developed a questionnaire, whether this is to assess food/nutrient intakes or for any other purpose, it is essential to carry out a feasibility study (pilot study). This should be done using similar subjects to those who will take part in the main study. The aims of the pilot study are to ensure that subjects will be able to answer the questions in a reasonable time and to identify any questions likely to pose difficulties for the main survey. The pilot study may be useful in identifying food items that are rarely consumed in the population and which could therefore be omitted from the questionnaire, reducing its length. It may also be helpful in identifying any additional foods consumed in the population which should be included in the questionnaire.

\section{VALIDATION OF INTAKE DATA}

If developing a questionnaire to estimate nutrient intakes, it is essential to assess the validity of the intake estimates for the population to be surveyed. Validity (the extent to which the questionnaire measures what is intended), i.e. usual intake, is particularly difficult since the true intake is unknown. However, "relative" validation exercises can be undertaken, i.e. validation of one method against another which has greater acceptance. The weighed inventory is often used to validate other dietary survey methods. It does, however, have disadvantages in that subjects may eat differently during the recording period or may fail to record all items eaten (Bingham, 1987). Another way to validate intake data is to compare the data with the results of biochemical tests. For example, a questionnaire to estimate vitamin $C$ intake could be validated by measurement of plasma or leukocyte ascorbic acid concentrations.

Whether a particular questionnaire may be considered valid will be largely dependent on the purpose of the study. For example, in case-control or intervention studies the mean intakes of groups are compared. The questionnaire must therefore be able to satisfactorily estimate group mean intakes of the nutrients of interest. Studies using a semi-quantitative food frequency questionnaire have been reported to yield similar results to those from 7-day weighed intake records (Fehily et al, 1987; Fehily et al, 1988a; Burr et al, 1989). 
In a cross-sectional study to investigate whether there are associations between diet and disease risk factors such as blood lipids or blood pressure, the dietary survey method chosen needs to be able to rank individuals by their intakes for the foods or nutrients of interest. Recording methods such as the weighed intake record or food diary may be more likely to detect associations than a food intake questionnaire. In the Caerphilly Heart Disease Study, for example, significant associations were detected between dietary variables and plasma lipids, lipoproteins and haemostatic factors when intakes were estimated from 7-day weighed intake records (Rogers et al, 1988; Fehily et al, 1988b), but many of these associations were much weaker and not statistically significant when intake estimates from semi-quantitative food frequency questionnaire were used. However, recording methods are not always superior: they would not be suitable for items consumed infrequently, e.g. fatty fish. Also, a food frequency questionnaire may be just as likely to detect associations (and be more cost-effective) if the nutrients of interest are present in a few, easily identifiable foods, e.g. vitamin C.

In prospective (cohort) studies, statistical analyses include division of subjects into tertiles or quintiles according to their nutrient intakes. The number of new disease events during the follow-up period is then calculated for each tertile or quintile of the nutrient intake distribution. One can then assess whether those in the top quintile of the intake distribution have a greater or lesser disease risk than those in the bottom quintile and whether there is a trend in disease risk with intake. For this type of study therefore, the questionnaire needs to be able to correctly classify individuals as low, moderate or high consumers of particular foods or nutrients.

The question then is how good does the classification need to be? Typical values for comparison between a semi-quantitative food frequency questionnaire and weighed food intake records are that about $68 \%$ of subjects are classified in the same or adjacent quintile and $2 \%$ classified in opposite quintiles. In this case, the true difference between the top and bottom quintile is $50 \%$ less than the observed difference (Fehily, 1993). This is because although the mean value for the total group is the same, true means for the bottom two quintiles are higher than estimated, and true means for the top two quintiles are lower than estimated, i.e. the slope is less steep as a result of misclassification. This will therefore reduce the ability of the study to detect diet-disease associations. When comparing intake estimates from a food intake questionnaire with those from another (better accepted) method, $80 \%$ of subjects would need to be classified in the same fifth of the distribution and none classified in opposite fifths in order to maintain the gradient of the slope from bottom to top quintile of nutrient intake (Fehily, 1993).

Another way of looking at this problem is to examine the effect of misclassification on estimates of relative risk of disease. Comparison of intake estimates from questionnaires with those from weighed intake records or food diaries have reported correlation coefficients of $0.3-0.5$ for energy, protein, fat and carbohydrate (Yarnell et al, 1983; Burr et al, 1989; Brunner et al, 2001; Masson et al, 2001). Willett (1990) showed that if the true relative risk attributable to a dietary variable is 1.5 , a correlation coefficient of 0.4 between the estimated intake and true intake will yield a slightly lower estimated relative risk of 1.2. If the true relative risk is 3.0, then the observed relative risk would be 1.6. Higher correlations between the estimated intake and true intake would produce smaller underestimates in the relative risk of disease.

\section{CONCLUSION}

When developing a questionnaire for a research study, it is important not only that the factors discussed are considered, but that that they are considered at the design stage of the study. This is essential to ensure that data collection and analysis can be carried out effectively and that resources required for the study are used efficiently. 


\section{REFERENCES}

Bingham, S.A. (1987) The dietary assessment of individuals: methods, accuracy, new techniques and recommendations. Nutr. Abstracts Rev. (Series A ) 57, 705-740.

Bingham, S. \& Day, K. (1987) Average portion weights of foods consumed by a randomly selected British population sample. Hum. Nutr.: Appl. Nutr. 41A, 258-264.

Brunner, E., Stallone, D., Juneja, M., Bingham, S. \& Marmot, M. (2001) Dietary assessment in Whitehall II: comparison of $7 \mathrm{~d}$ diet diary and food-frequency questionnaire and validity against biomarkers. Br. J. Nutr. 86, 405-414.

Burr, M.L., Fehily, A.M., Rogers, S., Welsby, E., King, S. \& Sandham, S. (1989) Diet and Reinfarction Trial (DART): design, recruitment and compliance. Eur. Heart J. 10, 558-567.

Crawley, H. (1994) Food portion sizes. London: HMSO

Fehily, A.M. (1993) Methods of food intake measurement: their uses and abuses. BNF Nutr. Bull. 18, 2533.

Fehily, A.M., Butland, B.K., Holliday, R.M. \& Yarnell, J.W.G. (1988a) Dietary studies in the Caerphilly Heart Disease Survey. Food Sci. Nutr. 42F, 77-78.

Fehily, A.M. \& Hopkinson, T. (1993) Do we need information about portion sizes to rank individuals by their nutrient intakes? J. Hum. Nutr. Diet. 6, 101-111.

Fehily, A.M., Yarnell, J.W.G., Bolton, C.H. \& Butland, B.K. (1988b) Dietary determinants of plasma lipids and lipoproteins: The Caerphilly Study. Eur. J. Clin. Nutr. 42, 405-413.

Fehily, A.M., Yarnell, J.W.G. \& Butland, B.K. (1987) Diet and ischaemic heart disease in the Caerphilly study. Hum. Nutr.: Appl. Nutr. 41A, 319-326.

Hunter, D.J., Sampson, L., Stampfer, M.J., Colditz, G.A., Rosner B. \& Willett, W.C. (1988) Variability in portion sizes of commonly consumed foods among a population of women in the United States. Am. J. Epidemiol. 127, 1240-1249.

Masson, L.F., McNeill, G., Tomany, J.O., Peace, H.S., Bolton-Smith, C. \& Grubb, D.A. (2001) Comparison of a semi-quantitative food frequency questionnaire with 4-day weighed diet records in Scottish men and women. Proc. Nutr. Soc. 60, 137A.

Nelson, M., Atkinson, A. \& Mayer, J. (1997) Food Portion Sizes. A Photographic Atlas. London: MAFF.

Rogers, S., Yarnell, J.W.G. \& Fehily, A.M. (1988) Nutritional determinants of haemostatic factors in the Caerphilly study. Eur. J. Clin. Nutr. 42, 197-205.

Willett, W. (1990) Nutritional Epidemiology. Monographs in Epidemiology and Biostatistics Volume 15. Oxford: Oxford University Press.

Yarnell, J.W.G., Fehily, A.M., Milbank, J.E., Sweetnam, P.M. \& Walker, C.L. (1983) A short dietary questionnaire for use in an epidemiological survey: comparison with weighed dietary records. Hum. Nutr.: Appl. Nutr. 37A, 103-112. 